\title{
COVID-19 ventilator barotrauma management: less is more
}

\author{
Brian Housman ${ }^{1}$, Adam Jacobi ${ }^{2}$, Andrea Carollo ${ }^{1}$, Tamar Nobel ${ }^{1}$, Corey Eber ${ }^{2}$, Samuel Acquah ${ }^{3}$, \\ Charles Powell ${ }^{3}$, Andrew Kaufman ${ }^{1}$, Dong-Seok Lee ${ }^{1}$, Daniel Nicastri ${ }^{1}$, Ardeshir Hakami ${ }^{1}$, \\ Kimberly Song ${ }^{1}$, Roopa Kohli-Seth ${ }^{4}$, Raja Flores ${ }^{1}$
}

${ }^{1}$ Thoracic Surgery Department, Icahn School of Medicine at Mount Sinai, Mount Sinai Health System, New York, NY, USA; ${ }^{2}$ Diagnostic, Molecular and Interventional Radiology, Icahn School of Medicine at Mount Sinai, Mount Sinai Health System, New York, NY, USA; ${ }^{3}$ Pulmonary, Critical Care and Sleep Medicine, Icahn School of Medicine at Mount Sinai, Mount Sinai Health System, New York, NY, USA; ${ }^{4}$ Surgery, Institute for Critical Care Medicine, Icahn School of Medicine at Mount Sinai, Mount Sinai Health System, New York, NY, USA

Contributions: I) Conception and design: B Housman, A Carollo, S Acquah, A Jacobi, R Flores; (II) Administrative support: R Flores., C Eber, S Acquah, C Powell, A Kaufman; (III) Provision of study materials or patients: B Housman, DS Lee, D Nicastri, A Hakami, K Song; (IV) Collection and assembly of data: B Housman, A Carollo, T Nobel, A Jacobi; (V) Data analysis and interpretation: B Housman, D Nicastri, A Hakami, K Song, R Kohli-Seth; (VI) Manuscript writing: All authors; (VII) Final approval of manuscript: All authors.

Correspondence to: Raja Flores, MD. Ames Professor of Cardiothoracic Surgery, Chairman, Department of Thoracic Surgery, Mount Sinai Health System, Icahn School of Medicine at Mount Sinai, One Gustave L. Levy Place, Box 1023, New York, NY 10029, USA. Email: raja.flores@mountsinai.org.

Background: COVID-19 patients requiring mechanical ventilation may develop significant pneumomediastinum and sub-cutaneous emphysema without associated pneumothorax (SWAP). Prophylactic chest tube placement or sub-fascial "blowholes" are usually recommended to prevent tension pneumothorax and clinical decline. Risk of iatrogenic lung injury and release of virus into the environment is high. Incidence and conservative management data of such barotraumatic complications during the COVID-19 pandemic are lacking.

Methods: All patients with mediastinal air and SWAP evaluated by the department of Thoracic Surgery at the Mount Sinai Hospital between March 30 and April 10, 2020 were identified. All patients without pneumothorax were treated conservatively with daily chest $x$-ray and observation. Three patients had prophylactic chest tube placement prior to the study period without thoracic surgery consultation.

Results: There were 29 cases of mediastinal air with SWAP out of 171 COVID positive intubated patients $(17.0 \%)$ who were treated conservatively. Patients were intubated for an average of 2.4 days before SWAP was identified. 12 patients (41\%) had improvement or resolution without intervention. Two patients progressed to pneumothorax 3 and 8 days following initial presentation. Both had chest tubes placed without incident before there were any changes in oxygenation, hemodynamics, supportive medications, or ventilator settings. There were 3 patients who had percutaneous tubes placed before the study period all of whom had significant worsening of their sub-cutaneous air and air leak.

Conclusions: Conservative management of massive sub-cutaneous emphysema without pneumothorax in COVID-19 patients is safe and limits viral exposure to healthcare workers. Placement of chest tubes is discouraged unless a definite sizable pneumothorax develops.

Keywords: COVID-19; barotrauma; thoracic surgery; radiology; pulmonary critical care

Submitted May 13, 2020. Accepted for publication Sep 11, 2020.

doi: 10.21037/atm-20-3907

View this article at: http://dx.doi.org/10.21037/atm-20-3907 


\section{Introduction}

Many COVID-19 patients admitted to critical care units require invasive mechanical ventilation $(1,2)$. Current management guidelines are based upon acute respiratory distress syndrome (ARDS) data from a variety of etiologies (3). Incidence and management data of COVID-19 specific ventilator associated pulmonary complications are lacking.

Ventilator induced barotrauma includes pneumomediastinum, pneumothorax, subcutaneous emphysema, and thoracic compartment syndrome (3). As such, the accepted treatment approach has been to place surgical or percutaneous chest tubes or sub-fascial "blowholes" as prophylactic measures against clinical decline $(4,5)$.

Since the onset of the current pandemic, there has been an increase in reports of intubated patients with mediastinal air and sub-cutaneous emphysema without associated pneumothorax (SWAP) $(6,7)$. COVID-19 patients represent a unique population at high-risk of iatrogenic lung injury and air leak from chest tube placement resulting in release of aerosolized virus endangering health care workers. We describe our non-invasive management at a large academic institution in New York City.

We present the following article in accordance with the STROBE reporting checklist (available at http://dx.doi. org/10.21037/atm-20-3907).

\section{Methods}

All patient consults by the Department of Thoracic Surgery at the Mount Sinai Hospital with mediastinal air and SWAP between March 30 and April 10, 2020 were identified. Patient demographics, treatments and outcomes data were abstracted in a de-identified manner as part of a quality initiative to develop a departmental protocol to address this clinical problem.

At the time of evaluation, every patient was already intubated, with ventilator settings per COVID management practices. All cases were diagnosed using portable chest $\mathrm{x}$-ray and confirmed with bedside ultrasound when possible. Due to the critical nature of these patients for transport, only three CT scans were performed. All patients were treated initially with conservative management, which included a daily chest $\mathrm{X}$-ray, and observation for changes in vital signs, ventilator parameters, and supportive infusions. No chest tubes were placed by thoracic surgery unless a pneumothorax developed on subsequent imaging. While the authors were unable to find a precise description of "massive" sub-cutaneous emphysema (SQE), we define it as involving $>30 \%$ of total body surface area as defined by the "rule of 9's" for burn victims. The study was conducted in accordance with the Declaration of Helsinki (as revised in 2013). Institutional review board approval was waived as the project was considered a quality improvement initiative for the care of COVID patients.

\section{Statistical analysis}

Summary statistics on included patients are presented as percentages and median and interquartile range (IQR) for categorical and continuous variables, respectively.

\section{Results}

Out of 171 total COVID positive intubated patients, 29 cases $(17 \%)$ of mediastinal air and SWAP were seen. A summary of patient clinical characteristics and outcomes is presented in Table 1.

There were 21 male and 8 female patients with median age of 55 (IQR, 42-63). There were 10 patients with no appreciable medical history. The average BMI was 30.8 with 15 patients $(51.7 \%)$ with obesity $(\mathrm{BMI}>30)$. Patients were intubated for an average of 2.4 (IQR, 1-4) days before SWAP was identified, though it was present on the postintubation films in 4 patients. At the time of evaluation, the average peak inspiratory pressure (PIP) was $32 \mathrm{~cm}$ $\mathrm{H}_{2} \mathrm{O}$ (range, 24-38), mean alveolar pressure was 21 (range, 15-24), and positive end expiratory pressure (PEEP) was 13 (range, 10-15).

There were no acute changes in hemodynamics, supportive medications, or ventilator settings before our evaluation that would have suggested an evolving or unstable process.

\section{Pneumomediastinum/SWAP diagnostic characteristics}

All patients had mediastinal air and 23 patients (79\%) had massive SQE involving at least the neck, supraclavicular spaces, arms, and chest on X-ray. Patients with pneumothorax on initial films were treated with chest tube placement and excluded from this study. A bedside ultrasound was also performed on each patient which confirmed "lung sliding" and "sand-on-the-shore" morphology in M-mode.

Seven patients $(24 \%)$ were read as having "small" or 
Table 1 Patient information

\begin{tabular}{|c|c|}
\hline Characteristic & Data \\
\hline \multicolumn{2}{|l|}{ Patient demographics } \\
\hline Total SWAP patients & 29 \\
\hline Male & $21(72.4 \%)$ \\
\hline Female & $8(27.6 \%)$ \\
\hline Age & 55 (IQR, 42-63) \\
\hline \multicolumn{2}{|l|}{ Past medical history } \\
\hline No past medical history & $10(34.5 \%)$ \\
\hline Hypertension & $8(27.6 \%)$ \\
\hline Asthma & $6(20.7 \%)$ \\
\hline COPD & $1(3.4 \%)$ \\
\hline Diabetes & $7(24.1 \%)$ \\
\hline Coronary artery disease & $2(6.9 \%)$ \\
\hline Obesity & $15(51.7 \%)$ \\
\hline Average BMI & 30.8 (IQR, 26.3-32.7) \\
\hline \multicolumn{2}{|l|}{ Clinical features and outcomes } \\
\hline PIP & 32 (range, 24-38) \\
\hline MAP & 21 (range, 15-24) \\
\hline PEEP & 13 (range, 10-15) \\
\hline Massive SQE & $23(79.3 \%)$ \\
\hline Sub-massive SQE & $6(20.7 \%)$ \\
\hline Improvement in SQE & $12(41.4 \%)$ \\
\hline Progression to pneumothorax & $2(6.9 \%)$ \\
\hline
\end{tabular}

SWAP, sub-cutaneous emphysema without associated pneumothorax; IQR, interquartile range; $\mathrm{PMH}$, past medical history; COPD, chronic obstructive pulmonary disease; BMI, body mass index; PIP, peak inspiratory pressure; MAP, mean airway pressure; PEEP, positive end expiratory pressure; SQE, sub-cutaneous emphysema.

"trace pneumothoraces" of either one or both lung fields (see Figure 1). In each case, these were small spaces that did not extend significantly into the lateral or apical lung fields. They were believed to be radiographic artifact from overlying skin or extrinsic compression of the parietal pleura by subcutaneous air. These findings were also observed and either resolved or remained stable in every patient.

Only three CT scans were performed, each revealing similar findings: severe interstitial disease consistent with COVID infection, widespread SQE, mediastinal air, pneumopericardium, and no evidence of pneumothorax (see Figure 2).

\section{Post-consultation outcomes}

Up to the time of publication, 12 patients $(41.4 \%)$ had improvement or resolution of their subcutaneous air without any special intervention; including any manipulation of the endotracheal tube or balloon. Ventilator settings were adjusted only in accordance with standard critical care and ARDSNet protocols and not with respect to the SWAP. No patient developed hemodynamic instability or rapid decline in oxygenation to suggest tension pneumothorax or decreased thoracic compliance.

In 3 patients, prior to the study period, percutaneous chest tubes were placed by the primary team before evaluation by Thoracic Surgery despite the absence of a pneumothorax. In each case, the observed subcutaneous emphysema and air leak dramatically worsened suggesting iatrogenic injury.

Two of the 29 patients developed pneumothoraces during their course of observation. In the first patient, the pneumothorax occurred after 3 days on the opposite side of a visualized "small apical" space, and chest tube placement did not improve previously seen subcutaneous air. In the second patient, a pneumothorax developed on day 8 . A surgical chest tube was placed, and the subcutaneous emphysema resolved almost completely by the next morning. Both cases were found on daily CXR, and were intervened upon safely in a controlled setting before there were any ventilatory or hemodynamic changes.

\section{Discussion}

Barotrauma presenting as mediastinal or subcutaneous emphysema is a known complication of mechanical ventilation in patients with ARDS (4). In the current COVID-19 pandemic, current guidelines recommend a similar ventilatory strategy to ARDS (2). In our institution in New York City where we have a high volume of ventilated patients on high PEEP, we have observed approximately $1 / 5^{\text {th }}$ of ventilated patients with mediastinal or subcutaneous air.

In non-intubated patients, mediastinal air, with or without subcutaneous emphysema, is often treated with observation. Cases are routinely diagnosed with CT scan and hospital courses are typically short and uneventful (8).

With intubated patients, decision making is more complicated and historically favors surgical intervention 

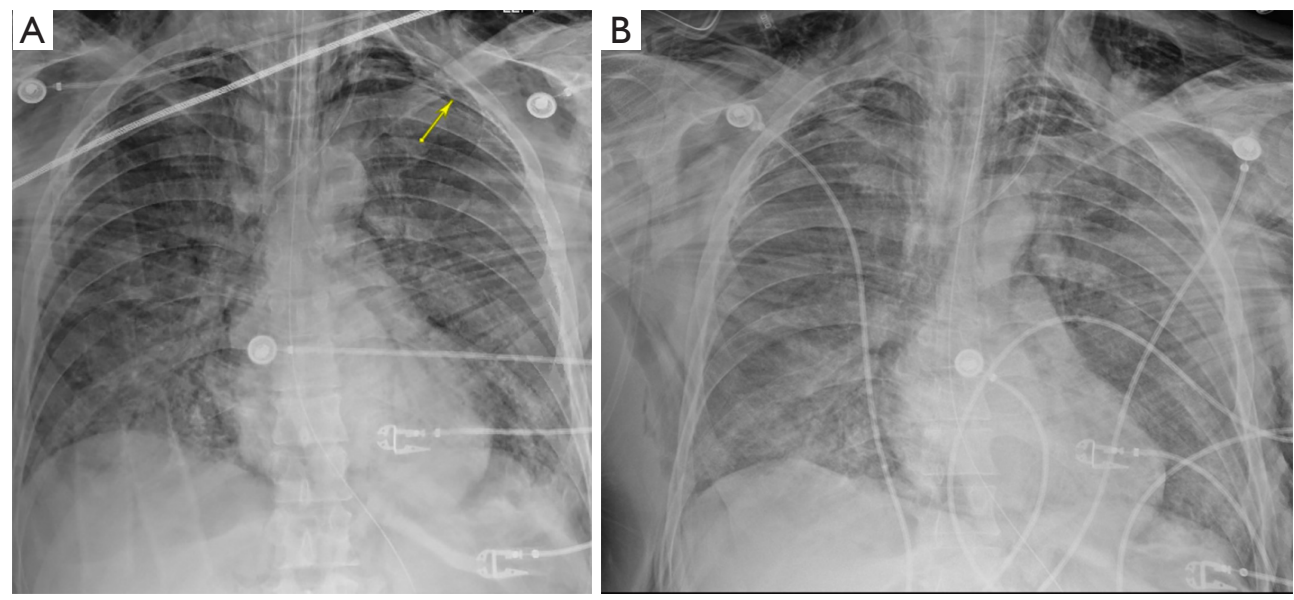

Figure 1 (A) Chest X-ray showing massive sub-cutaneous emphysema, read as having a "small apical pneumothorax." Note there is no component in the lateral aspect of the chest. (B) Radiograph of the same patient four hours later without a pneumothorax.

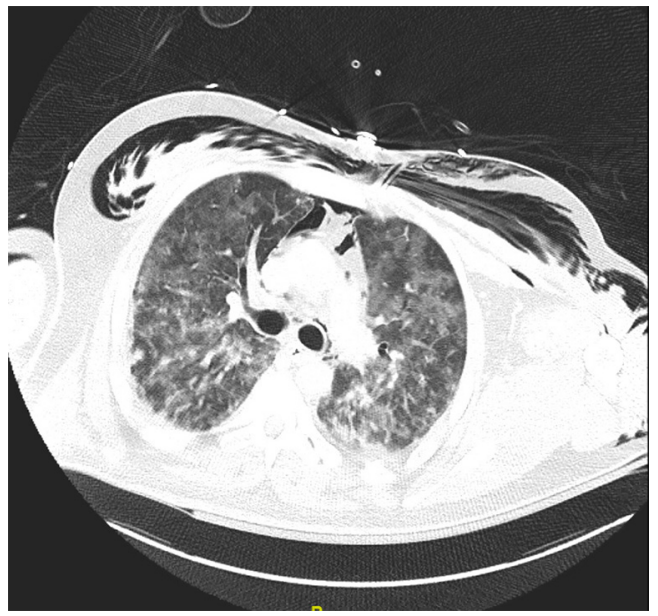

Figure 2 Characteristic CT of the Chest showing extensive bilateral ground-glass opacities, subcutaneous emphysema and mediastinal air, but no pneumothorax.

$(4,8)$. Positive pressure can worsen air deposits or exacerbate small parenchymal leaks. Over time, excessive subcutaneous pressure can theoretically result in decreased chest compliance and increased intra-thoracic pressure causing thoracic compartment syndrome (3). Tension pneumothoraces can also develop without obvious findings on imaging. Classically, this occurs when air is loculated in the anterior or deep sulcus spaces. Fortunately, when these occult processes are significant, they will still manifest changes in hemodynamics or oxygenation. A thorough clinical exam becomes an essential tool in determining the need for intervention. Unfortunately, since a majority of intubated COVID patients are hypoxic, receiving sedatives, vasopressors, paralytics, and are too unstable to be transported for CT imaging, we are often unable to evaluate these cases with conventional means.

Our series suggests that management of mediastinal air and SWAP without tube thoracostomy was not associated with hemodynamic instability or pulmonary compromise. We reasoned that in the absence of a pneumothorax, the SQE was likely the result of a mediastinal process. Pleural tubes, therefore, would be unable to drain the affected space, and would not reduce the spread of sub-cutaneous air.

Since SWAP patients have fully inflated lungs, tubes can only be safely placed with a surgical approach. In COVID-19 patients, even in experienced hands, this procedure carries a risk of iatrogenic injury, air leak with viral release, and the potential sequelae of large bore tubes on already fragile ventilatory dynamics (5). Additionally, per evolving protocols, many patients at the Mount Sinai Hospital are receiving therapeutic anticoagulation, and otherwise small traumatic lung hematomas may have significant consequences.

Sub-fascial "blowholes" can be placed with either negative pressure dressings or fenestrated angio-catheters (5). We have avoided these procedures since they increase the risk of widespread sub-dermal infection, and will endanger health care workers with a continuous release of aerosolized virus. Sub-cutaneous emphysema is a cosmetic problem until extreme pressures compromise hemodynamics or ventilation. This outcome is exceedingly rare, can be easily monitored in an ICU setting, and has never occurred in our patients.

Finally, small leaks that are missed by initial X-ray would 
eventually cause significant pneumothoraces. In the two cases presented earlier, the pneumothoraces were seen on a daily $\mathrm{X}$-ray, and intervened upon before the patients developed symptoms. Since the pneumothoraces manifested days after the initial presentation, there was speculation that both suffered a secondary barotrauma unrelated to the initial source of sub-cutaneous air.

At this time, we are unable to determine why $1 / 5^{\text {th }}$ of these patients are developing mediastinal air and SWAP. We can speculate multiple processes that may include traumatic intubation, tracheal mucosal dissection by high ventilator pressures, rupture of small sub-pleural blebs, or an etiology specific to the coronavirus (7).

\section{Conclusions}

Conservative management of massive subcutaneous emphysema without pneumothorax in COVID-19 patients is optimal for patient safety and limits viral exposure to healthcare workers. Placement of chest tubes is discouraged unless a definite sizable pneumothorax is present (6).

\section{Acknowledgments}

This study is dedicated in loving memory of Marg Oliver, Mount Sinai Thoracic Surgery OR technician.

Funding: None.

\section{Footnote}

Reporting Checklist: The authors have completed the STROBE reporting checklist. Available at http://dx.doi. org/10.21037/atm-20-3907

Data Sharing Statement: Available at http://dx.doi. org/10.21037/atm-20-3907

Conflicts of Interest: All authors have completed the ICMJE uniform disclosure form (available at http://dx.doi. org/10.21037/atm-20-3907). The authors have no conflicts of interest to declare.

Ethical Statement: The authors are accountable for all aspects of the work in ensuring that questions related to the accuracy or integrity of any part of the work are appropriately investigated and resolved. The study was conducted in accordance with the Declaration of Helsinki (as revised in 2013). Institutional review board approval was waived as the project was considered a quality improvement initiative for the care of COVID patients.

Open Access Statement: This is an Open Access article distributed in accordance with the Creative Commons Attribution-NonCommercial-NoDerivs 4.0 International License (CC BY-NC-ND 4.0), which permits the noncommercial replication and distribution of the article with the strict proviso that no changes or edits are made and the original work is properly cited (including links to both the formal publication through the relevant DOI and the license). See: https://creativecommons.org/licenses/by-nc-nd/4.0/.

\section{References}

1. Grasselli G, Zangrillo A, Zanella A, et al. Baseline Characteristics and Outcomes of 1591 Patients Infected With SARS-CoV-2 Admitted to ICUs of the Lombardy Region, Italy. JAMA 2020;323:1574-81.

2. Alhazzani W, Møller MH, Arabi YM, et al. Surviving Sepsis Campaign: guidelines on the management of critically ill adults with Coronavirus Disease 2019 (COVID-19). Intensive Care Med 2020;46:854-87.

3. Kukuruza K, Aboeed A. Subcutaneous Emphysema. 2020 Aug 8. In: StatPearls [Internet]. Treasure Island (FL): StatPearls, 2020.

4. Woodside KJ, vanSonnenberg E, Chon KS, et al. Pneumothorax in patients with acute respiratory distress syndrome: pathophysiology, detection, and treatment. J Intensive Care Med 2003;18:9-20.

5. Sciortino CM, Mundinger GS, Kuwayama DP, et al. Case report: treatment of severe subcutaneous emphysema with a negative pressure wound therapy dressing. Eplasty 2009;9:e1.

6. Zhou C, Gao C, Xie Y, et al. COVID-19 with spontaneous pneumomediastinum. Lancet Infect Dis 2020;20:510.

7. Ucpinar BA, Sahin C, Yanc U. Spontaneous pneumothorax and subcutaneous emphysema in COVID-19 patient: Case report. J Infect Public Health 2020;13:887-9.

8. Chu CM, Leung YY, Hui JY, et al. Spontaneous pneumomediastinum in patients with severe acute respiratory syndrome. Eur Respir J 2004;23:802-4.

Cite this article as: Housman B, Jacobi A, Carollo A, Nobel T, Eber C, Acquah S, Powell C, Kaufman A, Lee DS, Nicastri D, Hakami A, Song K, Kohli-Seth R, Flores R. COVID-19 ventilator barotrauma management: less is more. Ann Transl Med 2020;8(23):1575. doi: 10.21037/atm-20-3907 